\title{
Criticality and Big Brake singularities in the tachyonic evolutions of closed Friedmann universes with cold dark matter
}

\author{
Zsolt Horváth ${ }^{1}$, Zoltán Keresztes ${ }^{1,2}$, Alexander Yu. Kamenshchik ${ }^{3,4}$, László Á. Gergely ${ }^{1,2}$ \\ 1 Department of Theoretical Physics, University of Szeged, Tisza Lajos krt 84-86, Szeged 6720, Hungary \\ 2 Department of Experimental Physics, University of Szeged, Dóm Tér 9, Szeged 6720, Hungary \\ 3 Dipartimento di Fisica e Astronomia, Università di Bologna and INFN, via Irnerio 46, 40126 Bologna, Italy \\ 4 L.D. Landau Institute for Theoretical Physics, \\ Russian Academy of Sciences, Kosygin street 2, 119334 Moscow, Russia
}

\begin{abstract}
The evolution of a closed Friedmann universe filled by a tachyon scalar field with a trigonometric potential and cold dark matter (CDM) is investigated. A subset of the evolutions consistent to $1 \sigma$ confidence level with the Union 2.1 supernova data set is identified. The evolutions of the tachyon field are classified. Some of them evolve into a de Sitter attractor, while others proceed through a pseudo-tachyonic regime into a sudden future singularity. Critical evolutions leading to Big Brake singularities in the presence of CDM are found and a new type of cosmological evolution characterized by singularity avoidance in the pseudo-tachyon regime is presented.
\end{abstract}

PACS numbers: 98.80.-k, 98.80.Jk, 95.36.+x

\section{INTRODUCTION}

The discovery of the cosmic acceleration [1] has generated a lot of interest in Friedmann cosmological models with various kinds of exotic forms of matter, collectively known as dark energy [2]. Unsurprisingly, some of these models allow for novel type of singularities (occurring in finite time), which can be either strong or weak [3], [4], [5], 6].

In particular, the Big Rip singularity occurs at infinite scale factor, diverging Hubble parameter $H$ and diverging $\dot{H}$ [7], [8]. This singularity is strong according to both Tipler's [3] and Królak's [4] definition. Some other singularities are alike, but they occur at finite scale factor (FSF). The FSF singularity [8], [9] is also strong according to Królak's definition, but is classified as weak according to Tipler's definition.

The sudden future singularity (SFS), discovered many years ago [10], is characterized by both a finite scale factor and a finite Hubble parameter, while only $\dot{H}$ diverges [11]. As consequence the energy density is finite, while the pressure diverges at SFS. The geodesic equation (containing only $H$ ) remains regular at SFS, such that point particles will pass through the singularity. However for an infinitesimally short time the tidal forces in the geodesic deviation equation become infinite due to the diverging $\dot{H}$. The SFS singularity is weak in both Tipler's and Królak's definitions, hence it has been conjectured that finite object may also cross this singularity [5].

A subclass of the SFS singularities is the Big Brake, introduced in Ref. [12] as emerging in the presence of a particular tachyonic scalar field (i.e. Born-Infeld-type field). Such a singularity is characterized by a full stop in the expansion, occurring at finite scale factor and vanishing energy density, augmented by a diverging deceleration and pressure. There is also the w-singularity [8], [13], when both the pressure and energy density vanish, however their ratio, the barotropic index $w$ diverges, is also weak in both definitions.

Interesting features of the SFS singularities have been found on the example of the flat Friedmann universe filled with a combination of cold dark matter (dust) and tachyonic field, the latter obeying a particular potential of trigonometric functions [12]. The model possesses two surprising features. First, for certain model parameter range, at some stage of the cosmological evolution, the tachyonic field is constrained to transform into another Born-Infeld-type field, the pseudo-tachyon. Secondly, in this regime the universe unavoidably evolves into the SFS.

In this model in the absence of the dust component the SFS singularity represents a Big Brake. Confrontation with type Ia Supernovae (SNIa) data showed that the allowed cosmological evolutions can reach either the Big Brake or the de Sitter attractor [15]. Further, in Ref. [16] it was proven that the Big Brake singularity is traversable, and the evolution continues through a collapsing universe into a Big Crunch.

When the dust component is included, the SFS cannot be a Big Brake anymore [17]. Indeed, the universe reaches the singularity with a finite but nonzero $H$. Its traversability is guaranteed by certain changes in the matter properties [14], 18], imposed by the requirement of a smooth passage through the SFS. These changes lead to the notion of the quasi-tachyonic Born-Infeldtype field, a phantom field [19] with negative energy density. A throughout confrontation of these models with SNIa, baryonic acoustic peaks, and cosmic microwave background temperature anisotropy power spectrum did not disrule the evolutions leading to either the de Sitter attractor or to the SFS [20].

The present work represents a continuation of the series of papers [12], [15]-[18], [20], investigating the consequences of the introduction of a tiny positive curvature, which remains compatible with the recent Planck data [21], 22]. As will be shown, the addition of the curva- 
ture term enriches the velocity phase space of the possible cosmological evolutions, adding to it regimes which were impossible to realize in the flat model. We study these regimes numerically and show the existence of a critical behavior in the formation of the singularities in the framework of these tachyonic cosmological models. Such critical phenomena were well-known in the numerical study of gravitational collapse of a scalar field 23] and of various other fields (as reviewed in Ref. [24]), with the critical evolutions separating black-hole reaching evolutions from those which do not lead to black hole formation.

The structure of the paper is as follows. In Section III we introduce the equations of the tachyonic cosmology in the presence of dust and curvature. In Section III we confront the luminosity distance-redshift relation with the supernova data. In Section IV we investigate numerically the dynamics and give the classification of all possible regimes, with emphasis on the new types of evolutions. The results are summarized in the Conclusions.

The units are chosen as $c=1$ and $8 \pi G / 3=1$.

\section{A MIXTURE OF DUST AND TACHYONIC FIELD IN A FRIEDMANN UNIVERSE}

We consider a Friedmann universe

$d s^{2}=d t^{2}-a^{2}(t)\left(\frac{1}{1-K r^{2}} d r^{2}+r^{2} d \theta^{2}+r^{2} \sin ^{2} \theta d \phi^{2}\right)$,

with cosmological time $t$, comoving radial distance $r$, polar and azimuthal angles $\theta$ and $\phi$, unnormalized curvature index $K>0,=0$ or $<0$ and scale factor $a(t)$. The combined energy momentum tensor is an ideal fluid with energy density $\rho$ and pressure $p$. The dynamics of the scale factor is governed by the Raychaudhuri equation:

$$
\dot{H}-\frac{K}{a^{2}}+\frac{3}{2}(\rho+p)=0,
$$

and the change in the energy density by the continuity equation:

$$
\dot{\rho}+3 H(\rho+p)=0 .
$$

The overdot denotes time derivative and $H \equiv \dot{a} / a$ is the Hubble parameter. The first integral of the system (2)(3) is the Friedmann equation:

$$
H^{2}+\frac{K}{a^{2}}=\rho .
$$

We assume that the Friedmann universe is filled with a mixture of tachyonic field and cold dark matter (CDM). The mixture of the two ideal fluid components (distinguished by $m$ and $T$ subscripts) is characterized by $\rho=\rho_{m}+\rho_{T}$ and $p=p_{T}$, where

$$
\rho_{m}=\frac{\rho_{m}^{*}}{a^{3}}
$$

with integration constant $\rho_{m}^{*}$. The evolution of the homogeneous tachyonic field $T(t)$ follows from the Lagrangian density 25]

$$
L_{T}=-\sqrt{-g} V(T) \sqrt{1-g^{t t} s^{2}},
$$

with $s=\dot{T}$. Variation with respect to the metric gives its energy density

$$
\rho_{T}=\frac{V(T)}{\sqrt{1-s^{2}}}
$$

and pressure

$$
p_{T}=-V(T) \sqrt{1-s^{2}} .
$$

As $w=p / \rho=s^{2}-1$, in principle the tachyonic field can mimic dark energy. The particular case of a trigonometric tachyonic potential [12]

$$
V=\frac{\Lambda \sqrt{1-(1+k) y^{2}}}{1-y^{2}},
$$

with

$$
y=\cos \left[\frac{3}{2} \sqrt{\Lambda(1+k)} T\right],
$$

$\Lambda>0$ and $-1<k<1$ model parameters further leads to interesting behavior, including a smooth passage into the $s>1$ regime and a sudden future singularity which can be passed through by geodesics which then bounce back into a recollapse [12]-[20].

Note that the system is invariant under the simultaneous changes $(y \rightarrow-y, s \rightarrow-s)$. This yields a double coverage of the dynamics in these velocity phase space variables.

\section{TEST WITH SUPERNOVAE DATA}

The evolution of the Friedmann universe is determined by the initial conditions $H_{0}=H(z=0), y_{0}=y(z=0)$, $s_{0}=s(z=0),(z$ is the redshift $)$, the present values of the cosmological parameters

$$
\Omega_{m}=\frac{\rho_{m}^{*}}{a_{0}^{3} H_{0}^{2}}, \Omega_{K}=-\frac{K}{a_{0}^{2} H_{0}^{2}},
$$

associated to the CDM and the curvature, and the model parameters $\Lambda, k$. The parameter $\Omega_{K}$ is constrained by observations to $95 \%$ confidence level: $-0.0065 \leq \Omega_{K} \leq$ 0.0012 by WMAP data [27] and $-0.019 \leq \Omega_{K} \leq 0.011$ by Planck data (see fifth column in Table 5 of [22]). In what follows we set $\Omega_{m}=0.315$ (supported by the cosmic microwave anisotropies measured by the Planck satellite [21], 22] $), k=0.44, H_{0}=70 \mathrm{~km} / \mathrm{sec} / \mathrm{Mpc}$ and we analyze both $\Omega_{K}=-0.0065$ and 0 (the former being compatible with $K>0$, the latter representing a spatially flat 
universe). The Friedmann equation (4) represents a constraint among $y_{0}, s_{0}$ and $\Lambda$, thus only the first two are varied. The confrontation of the tachyonic model with the Union 2.1 SNIa data set [26] through a $\chi^{2}$-test as described in Ref. [15] relies on fitting with the computed luminosity distance $\left(d_{L}\right)$-redshift relation. A dimensionless luminosity distance $\hat{d}_{L}=H_{0} d_{L}$ is given by

$$
\begin{aligned}
& \sqrt{-\Omega_{K}} \frac{\hat{d}_{L}}{1+z}=\chi(z) \text { for } K=0, \\
& \sqrt{-\Omega_{K}} \frac{\hat{d}_{L}}{1+z}=\sin (\chi(z)) \text { for } K>0,
\end{aligned}
$$

with

$$
\frac{d \chi(z)}{d z}=\frac{\sqrt{-\Omega_{K}}}{\hat{H}}
$$

$\left(\hat{H}=H / H_{0}\right)$ We show the fit on Fig 1, The contours refer to the $68.3 \%(1 \sigma)$ and $95.4 \%(2 \sigma)$ confidence levels.
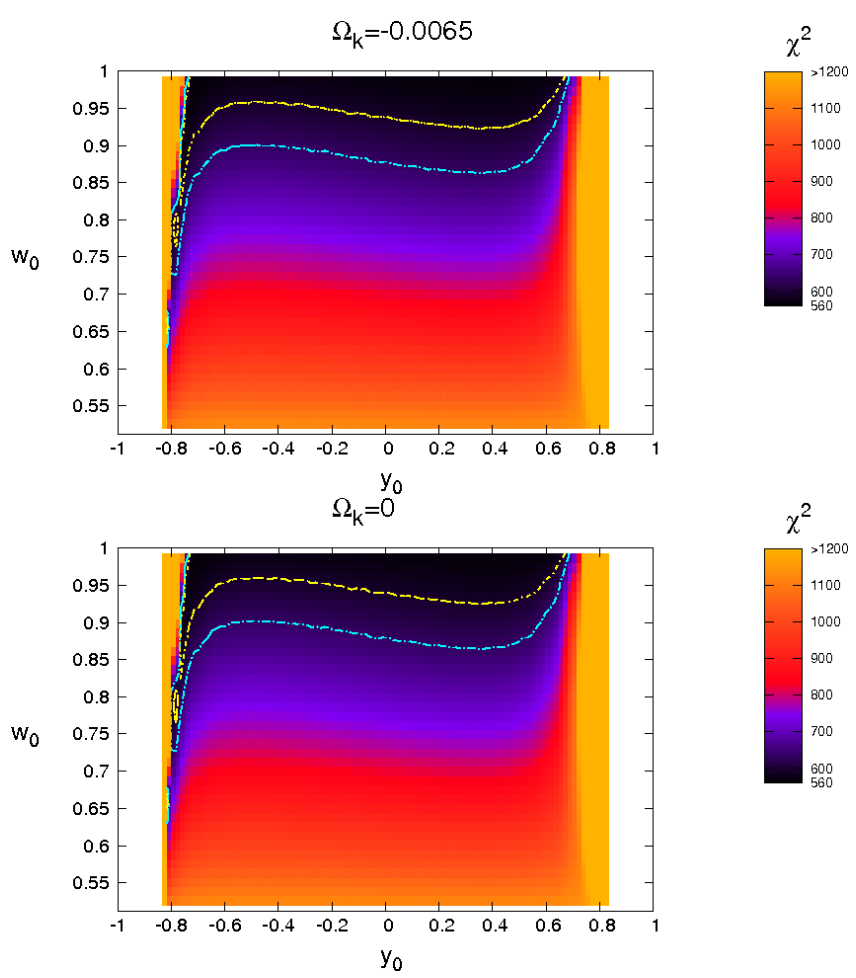

FIG. 1: (Color online) The fit of the luminosity distance vs. redshift for the parameters $k=0.44$ and $\Omega_{m}=0.315$ in the parameter plane $\left(y_{0}, w_{0}=\left(1+s_{0}^{2}\right)^{-1}\right.$ ) (upper panel for $\Omega_{K}=$ -0.0065 , lower panel for $\left.\Omega_{K}=0\right)$. The contours refer to the $1 \sigma$ and $2 \sigma$ confidence levels. The colour code of $\chi^{2}$ is indicated on the vertical stripes.

Thus the introduction of the curvature does not hamper the compatibility of the model with the SNIa observations.

\section{VELOCITY PHASE SPACE}

The $y^{2}<1 /(1+k), s^{2}<1$ domain of the velocity phase space represents proper tachyonic fields, characterized by the Lagrangian (6), energy density (7), pressure (8) and potential (9). A subset of the initial conditions $y_{0}, s_{0}$ allow the universe to evolve outside this regime through the corner points $y, s= \pm 1$ where the potential $V$ vanishes, as described in Ref. [12]. These are exceptional points, where the curvature does not diverge when $s^{2}=1$. When $s^{2}>1,(1+k)^{-1}<y^{2}<1$ the field becomes pseudo-tachyonic and $V$ imaginary. Although the Lagrangian (6) does not change in this regime, it is convenient to introduce the real potential

$$
W \equiv i V=\frac{\Lambda \sqrt{(1+k) y^{2}-1}}{1-y^{2}} .
$$

Note that in the Lagrangian $W$ absorbs the imaginary factor arising from the change of sign in the equation of state $w=s^{2}-1$. The expressions of the energy density and pressure are also unchanged in the pseudo-tachyonic regime. Rewriting the Lagrangian, the energy density and pressure in terms of $W$ however manifestly shows that all these quantities stay real:

$$
\begin{aligned}
L_{P} & =\sqrt{-g} W \sqrt{g^{t t} s^{2}-1}, \\
\rho_{P} & =\frac{W(T)}{\sqrt{s^{2}-1}} \\
p_{P} & =W(T) \sqrt{s^{2}-1} .
\end{aligned}
$$

The pressure is positive, thus the expansion of the universe slows down in this regime.

Further evolution to $s \rightarrow \pm \infty$ leads to SFS, arising for finite values of $z, \hat{H}$ and $W$ and $\rho_{P} \rightarrow 0, p_{P} \rightarrow \infty$. As was shown in Ref. [18] the evolution can be continued smoothly across these soft singularities at the price of a sudden change of the Lagrangian, which will represent a quasi-tachyonic field:

$$
\begin{aligned}
L_{Q} & =\sqrt{-g} W \sqrt{g^{t t} s^{2}+1}, \\
\rho_{Q} & =\frac{-W(T)}{\sqrt{s^{2}+1}} \\
p_{Q} & =W(T) \sqrt{s^{2}+1} .
\end{aligned}
$$

Note that $w_{Q}=p_{Q} / \rho_{Q}=-\left(s^{2}+1\right) \neq w$, thus the quasi-tachyon is a phantom with negative energy density.

In order to formulate the dynamical equations with dimensionless variables, we normalize the potentials as

$$
\begin{aligned}
\hat{V} & =\frac{\Omega_{\Lambda} \sqrt{1-(1+k) y^{2}}}{1-y^{2}}, \\
\hat{W} & =\frac{\Omega_{\Lambda} \sqrt{(1+k) y^{2}-1}}{1-y^{2}} .
\end{aligned}
$$

(i.e. $\Omega_{\Lambda}=\Lambda / H_{0}^{2}, \hat{V}=V / H_{0}^{2}, \hat{W}=W / H_{0}^{2}$ ). 
The dimensionless variables $z(t)$ and $\hat{H}(t)$ evolve as

$$
\begin{aligned}
\frac{d z}{d \hat{t}}+(1+z) \hat{H} & =0, \\
\frac{d \hat{H}}{d \hat{t}}+\frac{3}{2}\left(\hat{\rho}_{m}+\hat{\rho}+\hat{p}\right)+\Omega_{K}(1+z)^{2} & =0,
\end{aligned}
$$

with $\hat{t}=H_{0} t$. Eq. (24) follows from the definition of $z$, while Eq. (25) is the Raychaudhuri equation. Here $\hat{\rho}_{m}=\Omega_{m}(1+z)^{3}$ is the normalized density of the dust, while $\hat{\rho}$ stands for either of the normalized densities

$$
\begin{aligned}
& \hat{\rho}_{T}=\frac{\hat{V}}{\sqrt{1-s^{2}}}, \\
& \hat{\rho}_{P}=\frac{\hat{W}}{\sqrt{s^{2}-1}}, \\
& \hat{\rho}_{Q}=\frac{-\hat{W}}{\sqrt{s^{2}+1}},
\end{aligned}
$$

holding for the tachyonic, pseudo-tachyonic or quasitachyonic regimes, respectively. Similarly, $\hat{p}$ denotes one of the normalized pressures

$$
\begin{aligned}
& \hat{p}_{T}=-\hat{V} \sqrt{1-s^{2}}, \\
& \hat{p}_{P}=\hat{W} \sqrt{s^{2}-1}, \\
& \hat{p}_{Q}=\hat{W} \sqrt{s^{2}+1} .
\end{aligned}
$$

The dynamics of the tachyonic field $y(t)$ emerges from the definition of $s$ as

$$
\frac{d y}{d \hat{t}}+\frac{3}{2} s \sqrt{\Omega_{\Lambda}(1+k)\left(1-y^{2}\right)}=0,
$$

while the evolution of $s(t)$ in the tachyonic, pseudotachyonic and quasi-tachyonic regimes are given by the following Euler-Lagrange equations:

$$
\begin{aligned}
& \frac{d s}{d \hat{t}}+\left(1-s^{2}\right)\left(3 \hat{H} s+\frac{\hat{V}_{, T}}{\hat{V}}\right)=0, \\
& \frac{d s}{d \hat{t}}+\left(1-s^{2}\right)\left(3 \hat{H} s+\frac{\hat{W}_{, T}}{\hat{W}}\right)=0, \\
& \frac{d s}{d \hat{t}}+\left(1+s^{2}\right)\left(3 \hat{H} s-\frac{\hat{W}_{, T}}{\hat{W}}\right)=0 .
\end{aligned}
$$

In the neighborhood of the soft singularity $y$ can be approximated as

$$
\begin{aligned}
y_{P}= & y_{S}+\frac{\sqrt{6}}{2} \sqrt{1-y_{S}{ }^{2}} \\
& \times \sqrt{\Omega_{\Lambda}(1+k)} \sqrt{\frac{1}{H_{S}}} \sqrt{t_{S}-t}, \\
y_{Q}= & y_{S}-\frac{\sqrt{6}}{2} \sqrt{1-y_{S}^{2}} \\
& \times \sqrt{\Omega_{\Lambda}(1+k)} \sqrt{\frac{1}{H_{S}}} \sqrt{t-t_{S}} .
\end{aligned}
$$
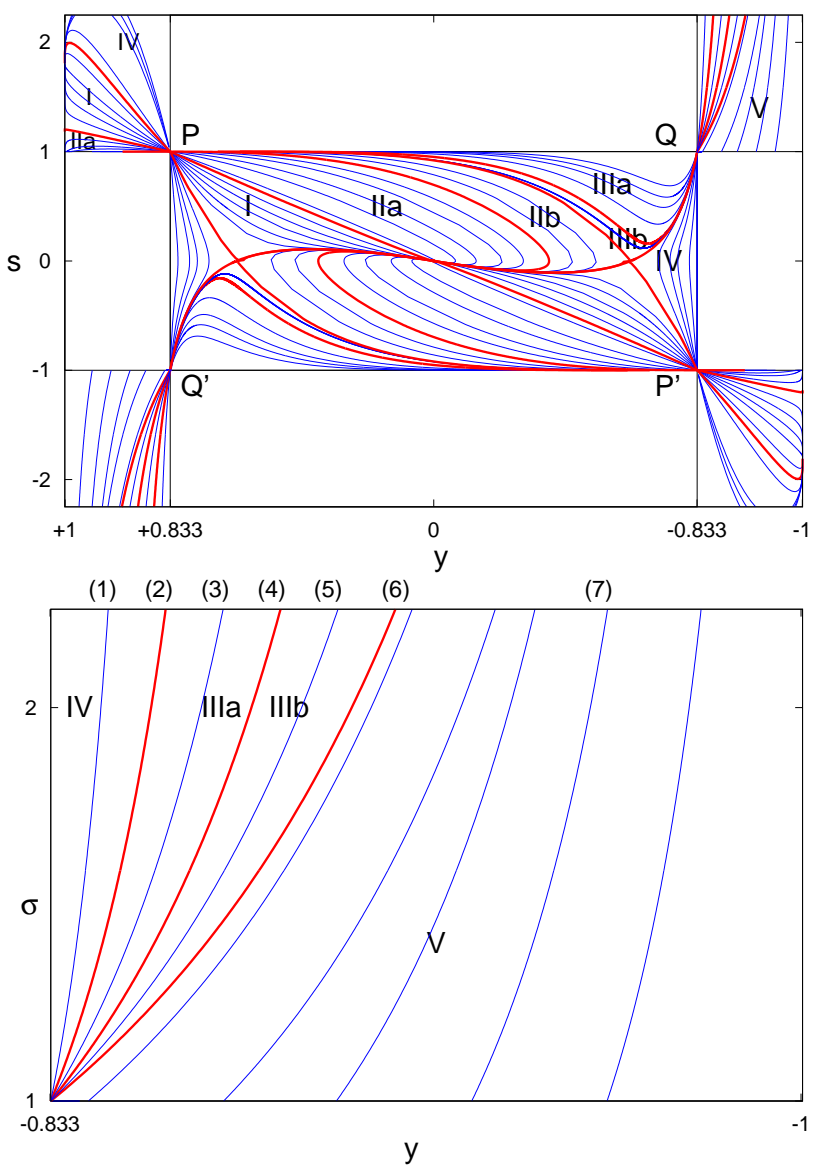

FIG. 2: (Color online) Upper panel: The numerical evolution of the dynamical equations yields seven type of trajectories (I, IIa, IIb, IIIa, IIIb, IV and V), separated by separatrices (red). Lower panel: the pseudo-tachyonic evolutions of the upper right corner. We chose $\Omega_{\Lambda}=0.8$.

(These formulas are analogous to Eqs. (30) and (50) of Ref. [18] presented in terms of the original tachyonic variable $T$, and they hold for the upper right strip of Fig. 2] where $y_{P}>y_{S}>y_{Q}$.) The two expressions coincide at the singularity. In the quasi-tachyonic regime the Hubble variable decreases, reaching zero at some point which is followed by a recollapse through the same type of singularity to the pseudo-tachyonic regime, ending in a Big Crunch. This was always the case for $K=0$ [16].

Due to the extra gravitational attraction represented by $\Omega_{K}<0$ however some of the pseudo-tachyonic evolutions do not reach the singularity, avoiding the quasitachyonic regime. Instead the recollapse into a Big Crunch commences earlier. These two types of evolutions are separated by a critical trajectory when the soft singularity is reached exactly with $\hat{H}=0$. In what follows we will investigate this criticality.

The numerical evolution of the dynamical equations yields seven types of trajectories, I, IIa, IIb, IIIa, IIIb, IV and V, represented on the upper panel of Fig. 2. The lower panel magnifies out the pseudo-tachyonic evo- 


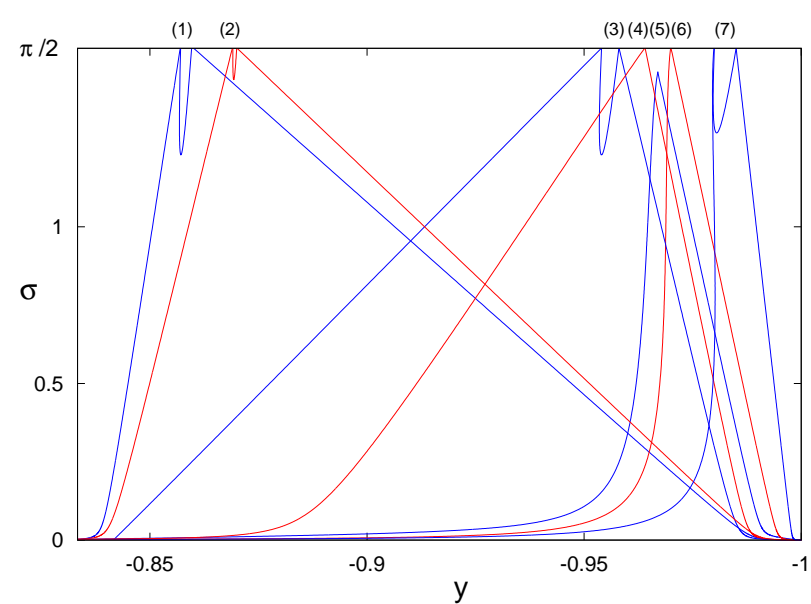

FIG. 3: (Color online) The trajectories presented on the lower panel of Fig. 2 are extended to infinity, and mapped into the interval $[0, \pi / 2]$ by a conformal transformation $\sigma=$ $\arctan \left(10^{-3} s\right)$. The pseudo-tachyonic and quasi-tachyonic regimes of each of type IV, IIIa, IIIb and V evolutions (blue), and the separatrices between types IV-IIIa, IIIa-IIIb and IIIb-V (red) are shown. The soft singularities correspond to $\sigma=\pi / 2$. The evolutions labeled by numbers are identical to those represented on the lower panel of Fig. 2. The critical evolutions leading to Big Brake singularities in the presence of dust are (4) and (6).

lutions of the upper right corner.

The trajectories of type I originate from the Big Bangs $(y, s)=(1, \sqrt{1+1 / k})$ and $(y, s)=(-1,-\sqrt{1+1 / k})$ in pseudo-tachyonic regime, then evolve into the tachyonic regime towards a de Sitter expansion, represented by the origin $(y, s)=(0,0)$. The trajectories of type IIa originate from the lines $s= \pm 1$ representing Big Bang singularities, then evolve from the pseudo-tachyonic regime, through the corner points into the tachyonic era, where they also reach the de Sitter attractor. The trajectories of type IIb are similar, but they never cross into the pseudo-tachyonic regime

The trajectories of type IIIa and IIIb also originate in Big Bangs at the lines $s= \pm 1$, pass through the tachyonic regime and through the cornerpoint into a pseudo-tachyonic regime, followed by a collapse into a Big Crunch (on the $y= \pm 1$ line). The difference between them is shown on Fig. 3. While IIIa reaches the soft singularity, evolves through a short quasi-tachyonic regime in which the universe start to recollapse towards a second soft singularity, those of type IIIb never reach a soft singularity.

The trajectories of type IV originate in a Big Bang located in the pseudo-tachyonic regime $(y= \pm 1$ line), evolve into the tachyonic regime, then return into a pseudo-tachyonic regime, pass twice through soft singularities (with a short quasi- tachyonic period), finally collapse into a Big Crunch. The trajectories of type V are born in the Big Bang singularities on the lines $s= \pm 1$, after which they mimic the evolution of the trajectories of type IV following their second passage into a pseudotachyonic regime.

On Fig. 3] we represent all types of trajectories which will reach a pseudo-tachyonic regime in the future (types IIIa, IIIb, IV and V). From among these IIIa, IV, V pass twice through soft singularities, with an intercalated quasi-tachyonic regime, while those of type IIIb never reach a soft singularity. We also determined numerically the critical evolutions separating these two types of behaviors. They are the evolutions leading to Big Brake type singularities in the presence of dust.

\section{CONCLUDING REMARKS}

In this paper we have expanded previous studies of a tachyonic cosmological model with trigonometric potential and dust exhibiting a rather rich spectrum of possible evolutions, by including curvature. As expected, a positive curvature could counterbalance the influence of the dust (manifesting itself in a positive $H$ and nonvanishing energy density at the SFS), in the sense that it can compensate for the nonzero energy density at the SFS. When the curvature term is strong enough, it stops the expansion before the encounter with the SFS, preventing its formation. If the curvature term is less strong, then the cosmological SFS is still formed, followed by the transition to the quasi-tachyonic regime, but its slowing-down effect is expected to reduce the duration of this regime as compared to the flat case.

We have shown by numerical methods that it is possible to have a particular balance between curvature and dust effects, leading to critical evolutions, represented by the curves (4) and (6) on Fig. 3. When such a critical evolution is realized, the universe reaches exactly a Big Brake singularity, which was forbidden in the flat case. The critical evolutions reaching a traversable Big Brake separate evolutions into SFS from completely regular ones. In this sense they resemble the criticality of singularity-forming in gravitational collapse. This criticality is a new feature of tachyonic cosmologies, induced by curvature. The possibility to reach a Big Brake type singularity when dust is present is the second new feature of the closed Friedmann universes with the tachyonic field.

\section{Acknowledgments}

We acknowledge stimulating interactions with David Polarski in the early stages of this work. Z. K. was supported by OTKA Grant No. 100216 and A. K. was partially supported by the RFBR Grant No. 14-02-00894. 
[1] A. Riess et al, Observational evidence from supernovae for an accelerating universe and a cosmological constant, Astron. J. 116, 1009 (1998);

S. Perlmutter et al, Measurements of Omega and lambda from 42 high redshift supernovae, Astrophys. J. 517, 565 (1999).

[2] R. R. Caldwell, R. Dave and P. J. Steinhardt, Cosmological Imprint of an Energy Component with General Equation of State, Phys. Rev. Lett. 80, 1582 (1998);

C. Armendariz-Picon, V. F. Mukhanov and P. J. Steinhardt, A Dynamical Solution to the Problem of a Small Cosmological Constant and Late-time Cosmic Acceleration, Phys. Rev. Lett. 85, 4438 (2000);

C. Armendariz-Picon, V. F. Mukhanov and P. J. Steinhardt, Essentials of k-essence, Phys. Rev. D 63, 103510 (2001);

T. Padmanabhan, Accelerated expansion of the universe driven by tachyonic matter, Phys. Rev. D 66, 021301 (2002);

A. Frolov, L. Kofman and A. Starobinsky, Prospects and Problems of Tachyon Matter Cosmology, Phys. Lett. B 545, 8 (2002);

L. Amendola, F. Finelli, C. Burigana and D. Carturan, WMAP and the Generalized Chaplygin Gas, J. Cosmol. Astropart. (JCAP) 07, 005 (2003);

H. Sandvik, M. Tegmark, M. Zaldarriaga and I. Waga, The end of unified dark matter?, Phys. Rev. D 69, 123524 (2004);

P. P. Avelino, L. M. G. Beca, J. P. M. de Carvalho, C. J. A. P. Martins and E. J. Copeland, The onset of the non-linear regime in unified dark matter models, Phys. Rev. D 69, 041301 (2004);

A. Dev, D. Jain and J. S. Alcaniz, Constraints on Chaplygin quartessence from the CLASS gravitational lens statistics and supernova data, Astron. Astrophys. 417, 847 (2004);

Z.-H. Zhu, Generalized Chaplygin gas as a unified scenario of dark matter/energy: observational constraints, Astron. Astrophys. 423, 421 (2004);

E. J. Copeland, M. R. Garousi, M. Sami and S. Tsujikawa, What is needed of a tachyon if it is to be the dark energy?, Phys. Rev. D 71, 043003 (2005);

R. Lazkoz, Rigidity of cosmic acceleration in a class of kessence cosmologies, Int. J. Mod. Phys. D 14, 635 (2005); V. Gorini, A. Kamenshchik, U. Moschella, V. Pasquier and A. Starobinsky, Stability properties of some perfect fluid cosmological models, Phys. Rev. D 72, 103518 (2005)

Cs. Csáki, N. Kaloper and J. Terning, The Accelerated Acceleration of the Universe, J. Cosmol. Astropart. Phys. (JCAP) 06, 022 (2006);

V. Sahni and A. A. Starobinsky, Reconstructing dark energy, Int. J. Mod. Phys. D 15, 2105 (2006);

E.J. Copeland, M. Sami, and S. Tsujikawa, Dynamics of dark energy, Int. J. Mod. Phys. D 15, 1753 (2006);

R. de Putter, E. V. Linder, Kinetic k-essence and Quintessence, Astropart. Phys. 28, 263 (2007);

C. S. J. Pun, L. Á. Gergely, M. K. Mak, Z. Kovács, G. M. Szabó, and T. Harko, Viscous dissipative Chaplygin gas dominated homogeneous and isotropic cosmological models, Phys. Rev. D 77, 063528 (2008);
R. J. Yang, S. N. Zhang and Y. Liu, Constraints on the generalized tachyon field models from latest observational data, J. Cosmol. Astropart. Phys. (JCAP) 01, 017 (2008);

O. Sergijenko, R. Durrer and B. Novosyadlyj, Observational constraints on scalar field models of dark energy with barotropic equation of state, J. Cosmol. Astropart. Phys. (JCAP) 08, 004 (2011);

S. Tsujikawa, Quintessence: A Review, Class. Quantum Grav. 30, 214003 (2013);

B. Novosyadlyj, O. Sergijenko, R. Durrer and V. Pelykh, Constraining the dynamical dark energy parameters: Planck-2013 vs WMAP9, J. Cosmol. Astropart. Phys. (JCAP) 05, 030 (2014);

[3] F. J. Tipler, Singularities in conformally flat spacetimes, Phys. Lett. A 64, 8 (1977).

[4] A. Królak, Towards the proof of the cosmic censorship hypothesis, Class. Quantum Grav. 3, 267 (1986).

[5] L. Fernández-Jambrina and R. Lazkoz, Geodesic behavior of sudden future singularities, Phys. Rev. D 70, 121503 (2004);

L. Fernández-Jambrina and R. Lazkoz, Classification of cosmological milestones, Phys. Rev. D 74, 064030 (2006).

[6] L.Á. Gergely, Z. Keresztes, and A. Yu. Kamenshchik, Distributional cosmological quantities solve the paradox of soft singularity crossing, AIP Conf. Proc. 1514, 132135 (2013).

[7] R.R. Caldwell, M. Kamionkowski, and N.N. Weinberg, Phantom Energy and Cosmic Doomsday, Phys. Rev. Lett. 91, 071301 (2003).

[8] S. Nojiri, S. D. Odintsov, and S. Tsujikawa, Properties of singularities in (phantom) dark energy universe, Phys. Rev. D 71, 063004 (2005).

[9] F. Cannata, A. Yu. Kamenshchik, and D. Regoli, Scalar field cosmological models with finite scale factor singularities, Phys. Lett. B 670, 241 (2009);

T. Denkiewicz, Observational constraints on finite scale factor singularities, J. Cosmol. Astropart. Phys. 07, 036 (2012);

A. Balcerzak and T. Denkiewicz, Density perturbations in a finite scale factor singularity universe, Phys. Rev. D 86, 023522 (2012).

[10] J. D. Barrow, G.J. Galloway, and F. Tipler, The closed universe recollapse conjecture, Mon. Nat. R. Astron. Soc. 223, 835 (1986).

[11] Yu. Shtanov and V. Sahni, Unusual cosmological singularities in brane world models, Classical Quantum Gravity 19, L101 (2002);

J. D. Barrow, Sudden future singularities, Classical Quantum Gravity 21, L79 (2004);

J. D. Barrow, More general sudden singularities, Classical Quantum Gravity 21, 5619 (2004);

S. Nojiri and S. D. Odintsov, Quantum escape of sudden future singularity, Phys. Lett. B 595, 1 (2004);

S. Nojiri and S. D. Odintsov, The final state and thermodynamics of dark energy universe, Phys. Rev. D 70, 103522 (2004);

M. P. Dabrowski, T. Denkiewicz, and M. Hendry, How far is it to a sudden future singularity of pressure?, Phys. Rev. D 75, 123524 (2007);

J. D. Barrow and S.Z.W. Lip, Classical Stability of Sud- 
den and Big Rip Singularities, Phys. Rev. D 80, 043518 (2009);

J. D. Barrow, A. B. Batista, G. Dito, J. C. Fabris and M. J. S. Houndjo, Sudden singularities survive massive quantum particle production, Phys. Rev. D 84, 123518 (2011);

T. Denkiewicz, M.P. Dabrowski, H. Ghodsi, M.A. Hendry, Cosmological tests of sudden future singularities, Phys. Rev. D 85, 083527 (2012);

J. D. Barrow and S. Cotsakis, General Dynamics of Varying-Alpha Universes, Phys. Rev. D 88, 067301 (2013);

T. Denkiewicz, Dark energy and dark matter perturbations in singular universes, arXiv:1411.6169 (2014).

[12] V. Gorini, A. Yu. Kamenshchik, U. Moschella, and V. Pasquier, Tachyons, scalar fields, and cosmology, Phys. Rev. D 69, 123512 (2004).

[13] M.P. Dabrowski and T. Denkiewicz, Barotropic index w-singularities in cosmology, Phys. Rev. D 79, 063521 (2009).

[14] A.Yu. Kamenshchik, Quantum cosmology and late-time singularities, Classical Quantum Gravity 30173001 (2013).

[15] Z. Keresztes, L.Á. Gergely, V. Gorini, U. Moschella, and A.Yu. Kamenshchik, Tachyon cosmology, supernovae data and the Big Brake singularity, Phys. Rev. D 79, 083504 (2009).

[16] Z. Keresztes, L.Á. Gergely, A.Yu. Kamenshchik, V. Gorini, and D. Polarski, Will the tachyonic universe survive the big brake?, Phys. Rev. D 82, 123534 (2010).

[17] Z. Keresztes, L.Á. Gergely, and A.Yu. Kamenshchik, Paradox of soft singularity crossing and its resolution by distributional cosmological quantities, Phys. Rev. D 86,
063522 (2012).

[18] Z. Keresztes, L.Á. Gergely, A.Yu. Kamenshchik, V. Gorini, and D. Polarski, Soft singularity crossing and transformation of matter properties, Phys. Rev. D 88, 023535 (2013).

[19] R. R. Caldwell, A Phantom Menace? Cosmological consequences of a dark energy component with supernegative equation of state, Phys. Lett. B 545, 23 (2002).

[20] Z. Keresztes and L.Á. Gergely, Combined cosmological tests of a bivalent tachyonic dark energy scalar field model, J. Cosmol. Astropartic. Phys. 11 (2014) 026.

[21] P. A. R. Ade et al. [Planck Collaboration], Planck 2013 results. XVI. Cosmological parameters, Astron. Astrophys. 571, A16 (2014).

[22] P. A. R. Ade et al. [Planck Collaboration], Planck 2015 results. XIII. Cosmological parameters, arXiv:1502.01589 (2015).

[23] M. W. Choptuik, Universality and scaling in gravitational collapse of a massless scalar field, Phys. Rev. Lett. 70, 9 (1993).

[24] C. Gundlach, J. M. Martín-García, Critical Phenomena in Gravitational Collapse, Living Rev. Relativity 10, 5 (2007).

[25] A. Sen, Rolling Tachyon, J. High Energy Phys. 04, (2002) 048.

[26] N. Suzuki et al., The Hubble Space Telescope Cluster Supernova Survey: V. Improving the Dark Energy Constraints Above $\mathrm{z}>1$ and Building an Early-Type-Hosted Supernova Sample, Astrophys. J. 746, 85 (2012).

[27] G. Hinshaw et. al., Nine-Year Wilkinson Microwave Anisotropy Probe (WMAP) Observations: Cosmological Parameter Results, Astrophys. J. Supp. 208, 19 (2013). 